\title{
Maduración de la Sutura Palatina Media En Adolescentes y Adultos Jóvenes Chilenos: Estudio Transversal.
}

\section{Midpalatal Sutural Maturation in Chilean Adolescents and Young Adults: Cross-sectional Study.}

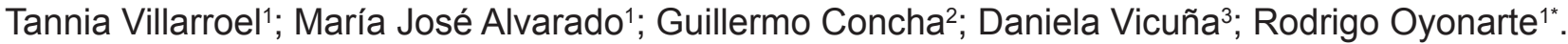

\author{
1. Departamento de Ortodoncia, Facultad de \\ Odontología, Universidad de los Andes, Santiago, \\ Chile. \\ 2. Departamento de Radiología Oral y Maxilofacial, \\ Facultad de Odontología, Universidad de los \\ Andes, Santiago, Chile. \\ 3. Departamento de Biomateriales Dentales, \\ Facultad de Odontología, Universidad de los \\ Andes, Santiago, Chile. \\ * Correspondencia Autor: Rodrigo Oyonarte. I \\ Direccion: Monseñor Álvaro del Portillo 12455, \\ Santiago, Las Condes, Región Metropolitana, Chile. \\ | E-Mail: royonarte@miuandes.cl \\ Trabajo recibido el 29/01/2020 \\ Trabajo revisado 08/09/2020 \\ Aprobado para su publicación el 08/11/2020
}

\begin{abstract}
RESUMEN
Objetivo: Evaluar la maduración de la sutura palatina media en adolescentes y adultos jóvenes chilenos, mediante valoración morfológica de imágenes de tomografía computarizada de haz cónico del maxilar. Material y método: Se analizó la sutura palatina media de 150 sujetos entre 15 y 30 años, seleccionados desde la base de datos radiológicos de un centro universitario de salud. La maduración sutural fue evaluada en el corte axial, utilizando el método de Angelieri y cols., clasificándola en cinco etapas (A, B, C, D y E). Se utilizaron las pruebas de correlación de Pearson para medir la concordancia intra e interexaminador, y T-Student para las diferencias entre sexos. Resultados: El estado de maduración más frecuente fue C $(43,3 \%)$, seguido por $E(33,3 \%)$ y D (22\%). En hombres, la etapa C fue más frecuente $(49 \%)$, mientras que en mujeres fue la etapa E (39\%). Sin embargo, no se encontraron diferencias estadísticamente significativas entre ambos sexos. Conclusiones: Aunque la mayoría de la muestra presentó una etapa tardía de maduración sutural, el $45 \%$ presentó estados de maduración sutural donde sería posible la expansión de naturaleza no quirúrgica. Debido a la variabilidad observada en adolescentes y adultos jóvenes, se recomienda la evaluación individual con imagenología 3D.
\end{abstract}

PALABRAS CLAVE

Suturas Craneales/Paladar Duro/ Crecimiento ; Desarrollo.

Int. J. Inter. Dent Vol. 14(2); 140-143, 2021.

\section{ABSTRACT}

Objective: To evaluate midpalatal suture maturation in Chilean adolescents and young adults through morphological assessment of cone-beam computed tomography images of the maxilla. Materials and methods: Analysis of the midpalatal suture of 150 subjects aged between 15 and 30 was performed, selected from a university clinical center radiological database. Sutural maturation was evaluated in the axial crosssection, using the method described by Angelieri et al., classifying it in five stages (A, B, $\mathrm{C}, \mathrm{D}$ and $\mathrm{E}$ ). Pearson's correlation tests were used to assess intra- and inter-examiner agreement, and T-Student for assessing differences between genders. Results: The most frequent maturation stage was C (43.3\%), followed by E (33.3\%) and D (22\%). In men, stage $C$ was more frequent $(49 \%)$, while in women it was stage $E(39 \%)$. However, no statistically significant differences were found between genders. Conclusions: Although the majority of the sample presented a late stage of sutural maturation, $45 \%$ presented states of sutural maturation where the expansion of a non-surgical nature would be possible. Due to the variability observed in adolescents and young adults, individual evaluation with 3D imaging is recommended.

\section{KEY WORDS}

Cranial Sutures/Palate; Hard/ Growth; Development.

Int. J. Inter. Dent Vol. 14(2); 140-143, 2021.

\section{INTRODUCCIÓN}

La expansión maxilar rápida (EMR) ha sido utilizada en la práctica ortodóncica para la corrección de problemas transversales, como mordidas cruzadas y resolución del apiñamiento dentario(1). Su objetivo es ampliar el maxilar mediante la separación de la sutura palatina media (SPM), gracias a la fusión tardía que presenta esta estructura ${ }^{(1,2)}$. Si bien este procedimiento se realiza con éxito en pacientes prepuberales, existe una gran variabilidad en los resultados clínicos en adolescentes y adultos jóvenes ${ }^{(3,4)}$. Esto se debe a que la edad cronológica no es confiable para determinar el estado de desarrollo de la SPM durante el crecimiento en estos pacientes ${ }^{(2,5)}$. Por esta razón, la evaluación individualizada de la maduración sutural en adolescentes y adultos jóvenes es considerada esencial, auxiliando en tomar decisiones terapéuticas, como sería realizar EMR con anclaje esquelético (MARPE) como alternativa menos invasiva a la expansión maxilar rápida quirúrgicamente asistida (SARPE) ${ }^{(6,7)}$

El reciente avance de las imágenes 3D, ha permitido una evaluación más detallada del desarrollo de estructuras craneofaciales sin la superposición de otras estructuras anatómicas. Angelieri y cols. ${ }^{(8)}$ propusieron un método de evaluación individual de la SPM mediante 
tomografía computarizada de haz cónico (CBCT), como una forma de proporcionar datos clínicos más confiables al momento de indicar MARPE o SARPE, metodología que hoy es utilizada extensamente.

Si bien existe evidencia del comportamiento de maduración de la SPM en la literatura, la heterogeneidad de las poblaciones en términos étnicos incorpora variabilidad y la información del nivel de maduración esperado en pacientes chilenos no está disponible. El grado de maduración de la SPM crea un desafío en la planificación del tratamiento y la obtención de resultados en la expansión maxilar, sobre todo en los pacientes adolescentes y adultos jóvenes. Debido a esto, el objetivo de esta investigación fue determinar el estado de maduración de la sutura palatina media a través de imágenes de CBCT en adolescentes y adultos jóvenes chilenos, de la comuna de San Bernardo, Región Metropolitana.

\section{MATERIALES Y MÉTODO}

Se realizó un estudio observacional, aprobado por el Comité de Investigación de la Facultad de Odontología de la Universidad de los Andes.

De acuerdo con los datos descritos por Angelieri y cols. ${ }^{(8,9)}$ se calculó el tamaño de la muestra utilizando las tablas de regresión logística de $H_{s i e h}^{(10)}$. Con un a de 0,05 y una potencia del $90 \%$, el número mínimo de individuos a incluir fue de 103. Se evaluaron 331 CBCT de maxilar superior obtenidos en el mismo centro radiológico entre enero 2015 y Julio 2018, los cuales fueron sometidos a criterios de inclusión y exclusión. Los criterios de inclusión comprendieron la edad, con sujetos de 15 a 30 años, y la utilización de imágenes de CBCT maxilares solicitadas con propósitos médicos. Fueron excluidas las imágenes de pacientes con anomalías o síndromes craneofaciales, o aquellas con artefactos o calidad deficiente para la evaluación.

Para determinar la calibración intra e interexaminador, se utilizó el índice de correlación de Pearson.

La muestra utilizada en el presente análisis incluyó 150 sujetos (73 hombres y 77 mujeres) de edades entre 15 a 30 años, cuyos detalles de distribución se presentan en tabla 2, con imágenes de CBCT, obtenidas retrospectivamente con formato DICOM, desde la base de datos del Centro Universitario de Especialidades Médicas y Odontológicas de la Universidad de los Andes, San Bernardo, Chile.

Las imágenes fueron obtenidas con un equipo radiológico (Ortophos XG3D Sirona, Alemania) con estandarización de la posición de cabeza y un campo de visión de $8 \times 8 \mathrm{~cm}$, tiempo de exposición de 14 segundos, tamaño del voxel de $0,16 \mathrm{~mm}, 85 \mathrm{kV}(\mathrm{p})$ y $7 \mathrm{~mA}$. Se utilizó el software Horos v1.1.7 para visualizar las imágenes.

La maduración de la sutura palatina media fue evaluada en el corte axial y clasificada utilizando un método de análisis visual de maduración de Angelieri y cols. ${ }^{(8)}$ (Figura 1, Tabla 1), por un examinador ciego y calibrado (T.V.D.), usando un computador con pantalla de alta definición en una habitación oscura. No se realizaron ajustes en el contraste o brillo de estas imágenes. Para la calibración, un radiólogo oral y maxilofacial experto y calibrado (G.C.S.), realizó la clasificación de 10 imágenes de cortes axiales, las cuales luego fueron presentadas al evaluador principal para su clasificación (T.V.D.). Se realizó posteriormente una segunda evaluación a los 10 días, donde las mismas imágenes fueron ordenadas al azar para su clasificación por el evaluador principal (T.V.D.).

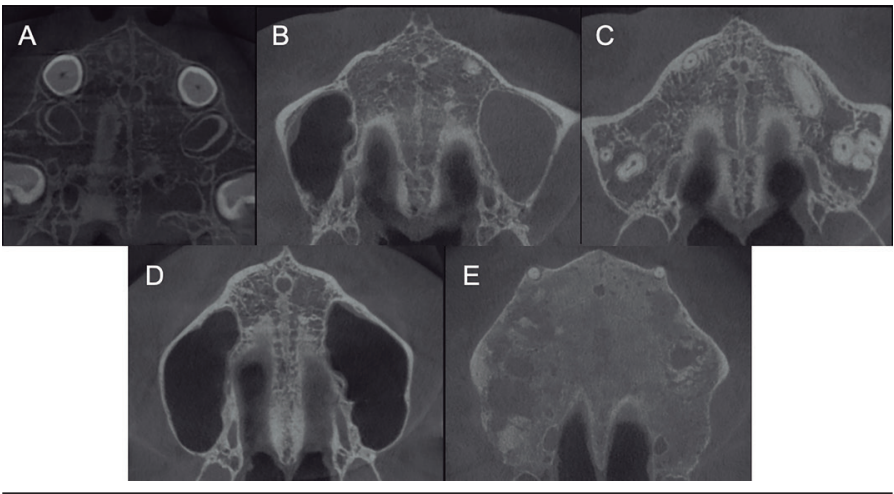

Figura 1. Etapas de maduración esqueletal de la sutura palatina media evaluadas. A, La sutura es visualizada como una línea hiperdensa relativamente recta, etapa $A$; $\mathbf{B}$, la sutura aparece como una línea festoneada de alta densidad, etapa B; C, dos líneas hiperdensas paralelas, festoneadas separadas por áreas de baja densidad, etapa C; D, los huesos palatinos se observan más hiperdensos y la sutura no puede ser visualizada en esta región, etapa D; E, no es posible visualizar la sutura a lo largo de los huesos maxilares y palatinos, etapa $\mathrm{E}$.
Tabla 1: Etapas de maduración esqueletal de la sutura palatina media según Angelieri y cols.

\begin{tabular}{c|l}
\hline Etapa & \multicolumn{1}{c}{ Descripción } \\
\hline A & $\begin{array}{l}\text { Línea sutural recta de alta densidad, sin o con poca } \\
\text { interdigitación. }\end{array}$ \\
\hline B & $\begin{array}{l}\text { Línea festoneada de alta densidad. Se pueden presentar } \\
\text { algunas pequeñas áreas donde hay dos líneas paralelas, } \\
\text { festoneadas, de alta densidad, separadas por pequeños } \\
\text { espacios de baja densidad. }\end{array}$ \\
\hline C & $\begin{array}{l}\text { Dos líneas paralelas, festoneadas, de alta densidad y } \\
\text { cercanas una de la otra, separadas por pequeños espa- } \\
\text { cios de baja densidad en el maxilar y el hueso palatino }\end{array}$ \\
\hline D & $\begin{array}{l}\text { Dos líneas paralelas, festoneadas, de alta densidad en la } \\
\text { porción maxilar del paladar, que no puede ser visualizada } \\
\text { en la porción palatina }\end{array}$ \\
\hline E & La sutura media palatina no puede ser visualizada. \\
\hline
\end{tabular}

\section{Análisis estadístico:}

Se realizó el test de Shapiro Wilk, estableciéndose la presencia de distribución normal de la muestra. Para el análisis de los resultados se utilizó la prueba T-Student para determinar diferencias entre sexo y clasificación sutural, y ANOVA con test de comparaciones múltiples de Bonferroni para determinar diferencias entre clasificación sutural, sexo y grupo etario.

El análisis se realizó mediante el software estadístico SPSS 25, IBM y se fijó la significación estadística en p<0,05.

\section{RESULTADOS}

Los coeficientes de correlación de Pearson intra e interexaminador fueron de 0,944 ( $p<0,00$, IC 95\%) y 1,0, respectivamente. La distribución de las etapas de maduración sutural según sexo y grupo etario de la muestra analizada son presentados en la Tabla 2.

El estado de maduración más frecuentemente encontrado en este estudio fue C (43,3\%), seguido por E (33,3\%) y D (22\%). En hombres, la etapa $C$ fue la más prevalente, con un $49,3 \%$, seguido por $E$ y $D(27,4 \%$ y $21,9 \%$ respectivamente). En mujeres el estado más frecuente fue $E$ con un $38,9 \%$, seguido por C $(37,6 \%)$. Sin embargo, no se encontraron diferencias estadísticamente significativas en el estado de maduración sutural entre sexos $(p=0,112)$.

Respecto a su distribución según rangos etarios, la etapa de maduración sutural más prevalente en el grupo de 15 a 20 años fue C $(65,3 \%)$, presentando una disminución de su prevalencia con el incremento de la edad. En los grupos de edad desde los 26 a los 30 años, la prevalencia de etapas D y $\mathrm{E}$ representan el $70 \%$ de ese rango etario. Se encontraron diferencias estadísticamente significativas entre los grupos etarios $(p=0,001)$. Estas diferencias se dieron entre el grupo de 15 a 20 años y los grupos de 21 a 25 años $(p=0,007)$ y 26 a 30 años $(p=0,002)$.

\section{DISCUSIÓN}

En la práctica clínica, todavía no existe consenso sobre los límites de edad para el tratamiento de los problemas transversales mediante la expansión maxilar rápida ${ }^{(3,8)}$. Se ha descrito que la SPM presenta gran variabilidad en su maduración esqueletal en relación con la edad y el sexo ${ }^{(1,11,12)}$, donde se observa que la fusión de ésta ocurre en individuos que van desde los 15 a 19 años, y hasta más de los 70 años ${ }^{(2,5,12)}$.

Angelieri y cols..$^{(8)}$ propusieron una metodología para obtener un corte transversal de la SPM en CBCT y realizar su evaluación morfológica individual en el eje axial, clasificándola en 5 etapas: A, B, C, D y E. Estos autores reportaron una gran variabilidad en la distribución de las etapas de maduración sutural respecto a la edad cronológica. Su estudio incluyó 140 sujetos, con edades desde 5,6 a 58,4 años. En el grupo de 14 - 18 años, un $56 \%$ de los sujetos presentó etapas de maduración tempranas compatibles con una EMR no quirúrgica. Sin embargo, los pacientes adultos evaluados fueron agrupados como un solo conjunto (>18 años), impidiendo realizar una evaluación de este grupo específico. Lamentablemente, existe escasa evidencia, con muestras pequeñas y edades muy heterogéneas, sobre el estudio de la representación morfológica individual de la SPM en este grupo etario. Este puede 
Tabla 2: Distribución de las etapas de maduración sutural por sexo y grupo etario.

\begin{tabular}{|c|c|c|c|c|c|c|c|}
\hline \multirow[t]{2}{*}{ Etapa } & \multicolumn{2}{|c|}{$15-20$ años } & \multicolumn{2}{|c|}{ 21-25 años } & \multicolumn{2}{|c|}{ 26-30 años } & \multirow{2}{*}{$\begin{array}{l}\text { Total } \\
\mathrm{n}(\%)\end{array}$} \\
\hline & Hombre $n(\%)$ & Mujer n(\%) & Hombre $n(\%)$ & Mujer n(\%) & Hombre $n(\%)$ & Mujer n(\%) & \\
\hline B & 0 & 0 & $1(0,67)$ & $1(0,67)$ & 0 & 0 & $2(1,33)$ \\
\hline $\mathrm{C}$ & $18(12)$ & $14(9,33)$ & $9(6)$ & $9(6)$ & $9(6)$ & $6(4)$ & $65(43,33)$ \\
\hline Total & $24(16)$ & $25(16,66)$ & $26(17,33)$ & $25(16,67)$ & $23(15,34)$ & $27(18)$ & $150(100)$ \\
\hline
\end{tabular}

presentar variadas respuestas clínicas frente a EMR, y es precisamente a ellos a quienes se dirige la utilización de anclaje esqueletal (MARPE) para lograr la expansión como opción de tratamiento no quirúrgico(6,7). Por esta razón, el objetivo del presente estudio fue evaluar el estado de maduración de la SPM en adolescentes y adultos jóvenes chilenos.

Los resultados obtenidos fueron consistentes con los de Angelieri y cols. ${ }^{(8,9)}$. Se encontraron diferencias en las etapas de maduración más frecuentes entre sexos, resultado esperable debido a que la maduración esqueletal comienza antes en las mujeres ${ }^{(13,14)}$. Sin embargo, estas no fueron estadísticamente significativas.

Según los grupos de edad, el presente estudio encontró una diferencia estadísticamente significativa en la maduración sutural, particularmente entre el grupo más joven y el resto de ellos. En pacientes de 15 a 20 años, se observó una prevalencia de la etapa C de más del $60 \%$, frecuencia que disminuyó gradualmente en los grupos etarios mayores. Esta prevalencia se asemeja a lo encontrado en el estudio de Ladewig y cols. ${ }^{(15)}$, quienes reportaron la maduración sutural en una muestra de 112 sujetos brasileros de 16 a 20 años. Encontraron que el estado $C$ es el más frecuente $(44,6 \%)$, seguido por $E(24,1 \%)$ y $D(23,2 \%)$, donde específicamente en los adolescentes de 17 y 18 años, más de un 50\% presentó etapa $\mathrm{C}$. Además, se observó que la prevalencia de etapas A y B sobre los 18 años fue de tan sólo un 2,5\%, similar a lo encontrado en el presente estudio, donde no se observaron individuos en etapa A y sólo el $1,3 \%$ de la muestra se encontraba en etapa $B$.

La prevalencia de etapas más avanzadas de maduración ( $D$ y E) se observó en un $55 \%$ de la muestra estudiada, incrementándose a mayor edad. Esto se encuentra en concordancia con lo planteado por Angelieri y cols. ${ }^{(9)}$ al estudiar maduración sutural en adultos de 18 a 66 años, donde reportaron que la mayoría de los sujetos presentaron estados avanzados de maduración ( $D=31 \%$ y $E=58 \%$ ), mientras que sólo el $12 \%$ presentó una sutura no fusionada (etapa B o C). Los autores no encontraron una asociación significativa entre la edad cronológica o el sexo, con los estados de maduración de la SPM. Sin embargo, cabe destacar que la muestra se separó en dos grandes grupos, entre aquellos menores y mayores de 30 años, y de los 78 sujetos de la muestra, sólo 14 eran hombres.

En 2017, Tonello y cols. ${ }^{(16)}$ utilizaron la misma metodología, pero en una muestra de pacientes de 11 a 15 años, en quienes sería esperable una EMR exitosa. Los autores concluyeron que los estados A, B y C estaban presentes en un $76,2 \%$ de la muestra, lo que afirmaría la predictibilidad del método. En nuestra investigación, el estadio C también fue el más prevalente, en especial en el grupo más joven. Sin embargo, la maduración de la SPM es un proceso biológico y no cambia instantáneamente de una etapa a otra, pudiendo estar presente de forma parcial o a lo largo de toda la sutura. Dado lo anterior, es posible que otros factores también puedan estar involucrados en el éxito de la EMR asistida con miniimplantes en adultos, como lo son la densidad ósea ${ }^{(2)} y$ la fusión de las diferentes suturas circunmaxilares $(2,17,18,19)$.

En el 2019, Isfeld y cols. ${ }^{(20)}$ evaluaron la confiablidad y utilidad del método propuesto por Angelieri y cols. como predictor de resultados exitosos en EMR. Reportaron que la concordancia interexaminador fue sólo moderada a débil, en contraste con lo reportado por los autores de este método ${ }^{(8)}$. Lo anterior sugiere que el protocolo de clasificación puede ser sólo relativamente confiable. Esto concuerda con lo observado por Barbosa y cols. ${ }^{(21)}$ en el 2017, donde evaluaron la confiabilidad y reproductibilidad de este método en ortodoncistas y radiólogos orales y maxilofaciales. A pesar de la alta concordancia interexaminador obtenida en el presente estudio, se hace necesario considerar los resultados de las otras investigaciones reportadas ${ }^{(20,21)}$, por lo que la concordancia observada en la aplicación de este método de evaluación sutural sólo sería alta en presencia de operadores altamente entrenados.

Por último, cabe mencionar que con los avances recientes en las técnicas MARPE ${ }^{(6,7,22-24)}$, la EMR es ahora posible en muchos de estos pacientes, lo que abre la discusión sobre las variables que juegan un rol definiendo la ocurrencia de la expansión maxilar en adultos. Es posible que otras características anatómicas del hueso maxilar superior, además de la SPM, sean relevantes en la factibilidad clínica de la ejecución de expansiones maxilares en adultos, particularmente las características de otras estructuras óseas y suturas del tercio medio facial(24,25)

En este contexto, el presente estudio ha permitido obtener información valiosa sobre los estados de maduración sutural palatina encontrados en población chilena de la región metropolitana, en base a una muestra extensa y homogénea, que pueden resultar un aporte para la toma de decisiones clínicas

\section{CONCLUSIONES}

Las características de la muestra utilizada, en cuanto a tamaño y distribución por grupos de edad, aporta datos epidemiológicos relevantes sobre la maduración de SPM en nuestra población. Aunque la mayoría de la muestra presentó una etapa tardía de maduración sutural, el $45 \%$ del total de la muestra presentó etapas intermedias de maduración donde es posible la expansión sin asistencia quirúrgica.

Existe una diferencia estadísticamente significativa en la maduración sutural, dependiente de la edad, donde los sujetos de 15 a 20 años presentan suturas medias palatinas más inmaduras, con una prevalencia de la etapa $\mathrm{C}$ de más del $60 \%$, frecuencia que disminuye gradualmente en los grupos etarios mayores.

Las etapas de maduración $\mathrm{D}$ y $\mathrm{E}$ son etapas donde la fusión sutural ha tenido lugar parcial o completamente. En este estudio, estas etapas fueron más frecuentes a medida que la edad aumenta, pero estuvieron presentes en todos los grupos etarios estudiados, con una prevalencia general del $55 \%$. Debido a la variabilidad observada en adolescentes y adultos jóvenes, se recomienda la evaluación individual con CBCT.

\section{CONFLICTO DE INTERESES}

Los autores declaran no tener conflictos de interés respecto de esta publicación 


\section{Bibliografía}

1. Bishara SE, Staley RN. Maxillary expansion: clinical implications. Am J Orthod Dentofacial Orthop. 1987;91:3-14.

2. Korbmacher H, Schilling A, Püschel K, Amling M, Kahl-Nieke B. Age-dependent three-dimensional microcomputed tomography analysis of the human midpalatal suture. J Orofac Orthop. 2007;68:364-76.

3. Handelman CS, Wang L, BeGole EA, Haas AJ. Nonsurgical rapid maxillary expansion in adults: report on 47 cases using the Haas expander. Angle Orthod. 2000;70:129-44.

4. Suri L Taneja P. Surgically assisted rapid palatal expansion: a literature review. Am J Orthod Dentofacial Orthop. 2008:133:290-302.

5. Knaup B, Yildizhan F, Wehrbein H. Age-related changes in the midpalatal suture. A histomorphometric study. J Orofac Orthop. 2004;65:467-74

6. Carlson C, Sung J, McComb RW, Machado AW, Moon W. Microimplant-assisted rapid palatal expansion appliance to orthopedically correct transverse maxillary deficiency in an adult. Am J Orthod Dentofacial Orthop. 2016;149(5):716-28.

7. Brunetto DP, Sant'Anna EF, Machado AW, Moon W. Non-surgical treatment of transverse deficiency in adults using Microimplant-assisted Rapid Palatal Expansion (MARPE). Dent Press J Orthod. 2017;22:110-25.

8. Angelieri F, Cevidanes LHS, Franchi L, Gonçalves JR, Benavides E, McNamara JA. Midpalatal suture maturation: classification method for individual assessment before rapid maxillary expansion. Am J Orthod Dentofacial Orthop. 2013;144:75969.

9. Angelieri F, Franchi L, Cevidanes LHS, Gonçalves JR, Nieri M, Wolford LM, et al. Cone beam computed tomography evaluation of midpalatal suture maturation in adults. Int J Oral Maxillofac Surg. 2017;46:1557-61.

10. Hsieh FY. Sample size tables for logistic regression. Stat Med.1989;8(7):795802 .

11. Melsen B. Palatal growth studied on human autopsy material. A histologic microradiographic study. Am J Orthod. 1975;68:42-54.

12. Persson M, Magnusson BC, Thilander B. Sutural closure in rabbit and man: a morphological and histochemical study. J Anat. 1978;125:313-21.

13. Björk A. Sutural growth of the upper face studied by the implant method. Acta Odontol Scand. 1966;24:109-27.

14. Fishman LS. Radiographic evaluation of skeletal maturation. A clinically oriented method based on hand-wrist films. Angle Orthod. 1982;52:88-112.
15. Ladewig VM, Capelozza-Filho L, Almeida-Pedrin RR, Guedes FP, de Almeida Cardoso M, de Castro Ferreira Conti AC. Tomographic evaluation of the maturation stage of the midpalatal suture in postadolescents. Am J Orthod Dentofacial Orthop. 2018;153:818-24.

16. Tonello DL, Ladewig VM, Guedes FP, Ferreira Conti ACC, Almeida-Pedrin RR, Capelozza-Filho L. Midpalatal suture maturation in 11- to 15-year-olds: A cone-beam computed tomographic study. Am J Orthod Dentofacial Orthop. 2017:152:42-8.

17. da Silva Filho OG, Magro AC, Capelozza Filho L. Early treatment of the Class III malocclusion with rapid maxillary expansion and maxillary protraction. Am J Orthod Dentofacial Orthop. 1998;113:196-203.

18. Melsen B, Melsen $F$. The postnatal development of the palatomaxillary region studied on human autopsy material. Am J Orthod. 1982;82:329-42.

19. Jafari A, Shetty KS, Kumar M. Study of stress distribution and displacement of various craniofacial structures following application of transverse orthopedic forces-a three-dimensional FEM study. Angle Orthod. 2003;73:12-20.

20. Isfeld D, Flores-Mir C, Leon-Salazar V, LagravËre M. Evaluation of a nove palatal suture maturation classification as assessed by cone-beam computed tomography imaging of a pre- and postexpansion treatment cohort. Angle Orthod. 2019;89:252-261.

21. Barbosa NMV, Castro AC, Conti F, Capelozza-Filho L, Almeida-Pedrin RR Cardoso MA. Reliability and reproducibility of the method of assessment of midpalatal suture maturation: A tomographic study. Angle Orthod. 2019; 89(1):71-7. 22. Cantarella D, Dominguez Mompell R, Moschik C, Mallya SM, Pan HC, Alkahtan MR, et al. Midfacial changes in the coronal plane induced by microimplant supported skeletal expander, studied with cone beam computed tomography images. Am J Orthod Dentofacial Orthop. 2018; 154(3): 337-45

23. Lin L, Ahn HW, Kim SJ, Moon SC, Kim SH, Nelson G. Tooth-borne vs bone-borne rapid maxillary expanders in late adolescence. Angle Orthod. 2014;85(2):253-62.

24. MacGinnis M, Chu H, Youssef G, Wu KW, Machado AW, Moon W. The effects of micro implant assisted rapid palatal expansion (MARPE) on the nasomaxillary complex a finite element method (FEM) analysis. Prog Orthod. 2014;15(1):52.

25. Cantarella D, Dominguez Mompell R, Mallya SM, Moschik C, Pan HC, Miller J, et al. Changes in the midpalatal and pterygopalatine sutures induced by micro implant supported skeletal expander, analyzed with a novel 3D method based on CBCT imaging. Prog Orthod. 2017;18(1):34. 